\title{
Cosmic ray transport in anisotropic magnetohydrodynamic turbulence
}

\section{Shear Alfvèn waves}

\author{
A. Teufel ${ }^{1}$, I. Lerche ${ }^{2}$, and R. Schlickeiser ${ }^{1}$ \\ ${ }^{1}$ Institut für Theoretische Physik, Lehrstuhl IV: Weltraum- und Astrophysik, Ruhr-Universität Bochum, 44780 Bochum, \\ Germany \\ e-mail: andreasm4@yahoo.com \\ ${ }^{2}$ Department of Geological Sciences, University of South Carolina, Columbia, SC 29208, USA \\ e-mail: black@geol.sc.edu
}

Received 31 May 2002 / Accepted 23 October 2002

\begin{abstract}
Observations of interstellar turbulence imply that the power spectrum of the wave turbulence must be highly anisotropic. This anisotropy has to be included when transport of high energy cosmic rays in the Galaxy is discussed. Here we evaluate the relevant cosmic ray transport parameters in the presence of anisotropic shear Alfvèn wave turbulence, which have no contribution from the transit-time damping interaction and are entirely due to gyroresonant interactions. By averaging the respective Fokker-Planck coefficient over the particle pitch-angle we calculate the momentum and spatial diffusion coefficients for different anisotropy parameters. For strongly perpendicular turbulence $(\Lambda \ll 1)$ we obtain that the momentum diffusion coefficient is proportional to $\Lambda^{(s+1) / 2}$, whereas for strongly parallel turbulence $(\Lambda \gg 1)$ the momentum diffusion coefficient is a constant. We also calculate the anisotropy dependence of the spatial diffusion coefficient and the mean free path for pure Alfvèn turbulence. For all coefficients we discuss the rigidity dependence for different cosmic ray particles and compare with earlier calculations of transport parameters for slab-type Alfvèn turbulence.
\end{abstract}

Key words. magnetohydrodynamics (MHD) - plasmas - turbulence - cosmic rays - ISM: magnetic fields

\section{Introduction}

Observations of interstellar scintillations (Rickett 1990; Spangler 1991), general theoretical considerations (Goldreich \& Sridhar 1995), and comparison of interstellar radiative cooling in HII-regions and in the diffuse interstellar medium with linear Landau damping estimates for fast-mode decay (Lerche \& Schlickeiser 2001a), all strongly imply that the power spectrum of wave turbulence in the interstellar medium must be highly anisotropic. It is not clear from the observations whether the turbulence spectrum is oriented mainly parallel or mainly perpendicular to the ambient magnetic field, either will satisfy the needs of balancing wave damping energy input against radiative cooling. This anisotropy must be included when transport of high energy cosmic rays in the Galaxy is discussed (Chandran 2001; Yan \& Lazarian 2002).

In the first paper of this series (Lerche \& Schlickeiser $2001 \mathrm{~b}$ - hereafter referred to LS) we have started to evaluate the relevant cosmic ray transport parameters in the presence of anisotropic fast magnetosonic plasma wave turbulence. All technical details of the calculation of Fokker-Planck coefficients and their relation to the transport parameters of the

Send offprint requests to: A. Teufel,

e-mail: ate@tp4.ruhr-uni-bochum.de cosmic ray diffusion-convection equation (as the parallel mean free path, the rate of adiabatic deceleration and the momentum diffusion coefficient) were presented there (see LS Sects. 2 and 3). However, in order to calculate these transport parameters in the interstellar medium we have to investigate (analogously to the analysis in LS for fast magnetosonic waves) also the influence of the anisotropy parameter on the Fokker-Planck coefficients for shear Alfvèn waves, because interstellar plasma turbulence most probably is a mixture of fast magnetosonic waves and shear Alfvèn waves. This analysis is the subject of the present paper. In order to avoid unnecessary repititions we will use the same notation and will frequently refer to equations in paper $L S$.

As LS we base our analysis on the quasilinear approximation. Because of the complicated nonlinear equations of motion of charged particles in partially random electromagnetic fields there are only two methods to study theoretically particle acceleration: (i) numerical simulations of highly idealized configurations, and (ii) quasilinear theory. Both have their advantages and shortcomings, and they complement each other. The quasilinear approach to the interaction of charged particles with parially random electromagnetic fields $\left(B_{0}+\delta B, \delta E\right)$ is a first-order perturbation calculation in the ratio $q_{\mathrm{L}}=\left(\delta B / B_{0}\right)^{2}$ and requires smallness of this ratio with respect to unity. 
Obviously, besides limited computer power numerical simulations require the precise knowledge of many important input plasma parameters as well as the specification of initial and boundary conditions which at least for the more distant cosmic objects are not known. By chosing the wrong input plasma quantities one may end up in an irrelevant range of solution space. Of course, when all these input quantities are known and given, the simulations result in a very accurate and detailed description of the acceleration processes on all spatial, momentum and time scales of interest.

On the other hand, the discussion of the accuracy of quasilinear theory is legendary in the literature of theoretical plasma physics especially in its application to plasma fusion devices. The quasilinear approach to the interaction of charged particles with parially random electromagnetic fields $\left(B_{0}+\delta B\right.$, $\delta E)$ is a first-order perturbation calculation in the ratio $q_{\mathrm{L}}=$ $\left(\delta B / B_{0}\right)^{2}$ and requires smallness of this ratio with respect to unity. More quantitaively, in the context of astrophysical cosmic ray propagation studies Michalek \& Ostrowski (1996) have compared the quasilinear values of cosmic ray transport parameters with the results of Monte Carlo simulations of the cosmic ray transport. They calculated numerically the trajectories in phase space of relativistic cosmic ray particles in an uniform magnetic field of strength $B_{0}$ with superposed plane parallel propagating Alfvèn waves of total magnetic field strength $\delta B$. By averaging over these trajectories the values of the transport parameters were derived. The calculations were performed for various wave amplitudes $\left(\delta B / B_{0}\right)^{2}$ ranging from 0.225 to 4.0 for a Kolmogorov turbulence spectrum. Over the whole range of wave amplitudes the agreement between simulated and quasilinear values of the cosmic ray transport parameters is remarkably good, and within factors of order 2 even at large amplitudes. These calculations demonstrate that, for small-amplitude $\left(\left(\delta B / B_{0}\right)^{2} \leq 4\right)$ plasma wave fields, quasilinear theory provides a reasonable description of cosmic ray transport.

From careful measurements of the interstellar turbulence Spangler (1991) as well as Minter \& Spangler (1997) estimate the mean of the interstellar magnetic field fluctuations due to turbulence of $\delta B \simeq 0.9 \mu \mathrm{G}$. Combined with studies of the rotation and dispersion measure of 185 pulsars (Lyne \& Smith 1989) that indicate an average field strength of about $2-3 \mu \mathrm{G}$, it seems that for the interstellar medium the requirement of small turbulence amplitudes $\left(q_{\mathrm{L}} \leq 1\right)$ for the applicability of the quasilinear approximation is well fulfilled.

A second assumption for the applicability of the quasilinear approximation concerns the coherence scale of the fluctuating electromagnetic fields $l_{\mathrm{c}}$ which in our case is related to the plasma wave autocorrelation time $t_{\mathrm{A}}$, i.e. the time for relative phases of the waves to randomize due to the difference in wave phase velocities, through $l_{\mathrm{c}}=V_{\mathrm{A}} t_{\mathrm{A}}$. The wave autocorrelation time has to be smaller than the particle trapping time. The physical arguments for this condition have been discussed by e.g. Karimabadi et al. (1992). Because the quasilinear Fokker-Planck description of cosmic ray transport is based on the Markovian assumption, it is necessary to introduce a decay in the wave's autocorrelation function entering the Fokker-Plack coefficients in order to avoid their quasi-periodicity which would be in conflict with the Markovian assumption. Karimabadi et al. (1992) have discussed both linear and nonlinear processed providing the decays of correlation functions.

Probably the most relevant decay process for interstellar cosmic ray propagation is the presence of interstellar wave damping in the partially ionised diffuse intercloud medium at a rate $\Gamma=1 / t_{\mathrm{A}}=10^{-10} n_{\mathrm{H}} \mathrm{s}^{-1}$ (Kulsrud \& Pearce 1969; Ferriere et al. 1988). As has been shown by Schlickeiser \& Achatz (1993) plasma wave damping leads to Breit-Wignertype resonance broadening, and due to the overlapping of the broadened resonances in phase space the correlation functions decay to zero at times $t>t_{\mathrm{A}}=300 n_{\mathrm{H}}$ yrs avoiding their quasi-periodic behaviour. Therefore a necessary condition for the validity of interstellar quasilinear cosmic ray transport parameters is $t>t_{\mathrm{A}}$ which is equivalent to the condition that the derived scattering mean free path $\lambda>l_{\mathrm{c}}=V_{\mathrm{A}} t_{\mathrm{A}} \simeq$ $3 \times 10^{16}\left(V_{\mathrm{A}} / 30 \mathrm{~km} \mathrm{~s}^{-1}\right) n_{\mathrm{H}}=0.01 \mathrm{pc}$ is larger than the coherence scale. Phenomenologically inferred estimates of the interstellar cosmic ray scattering length from the cosmic ray lifetime measurements indicate values of $\lambda \simeq 1 \mathrm{pc}$ so that this additional condition also is fulfilled.

\section{Cosmic ray Fokker-Planck coefficients for shear Alfvèn waves}

Adopting the same anisotropic magnetic turbulence tensor (Eqs. LS(10)-LS(12)) the three relevant quasilinear FokkerPlanck coefficients for shear Alfvèn waves with vanishing magnetic helicity are (Schlickeiser 2002, Ch. 13.1)

$$
\begin{aligned}
\left(\begin{array}{c}
D_{\mu \mu} \\
D_{\mu p} \\
D_{p p}
\end{array}\right) & =\frac{2 \pi^{2} \Omega^{2}\left(1-\mu^{2}\right)}{B_{0}^{2}} \sum_{j= \pm 1} I_{0}^{j} \sum_{n=-\infty}^{+\infty}\left(\begin{array}{c}
(1-j \mu \epsilon)^{2} \\
j \epsilon p(1-j \mu \epsilon) \\
(\epsilon p)^{2}
\end{array}\right) \int_{-1}^{+1} \mathrm{~d} \eta \\
& \times \frac{1+\eta^{2}}{\left[\eta^{2}+\Lambda\left(1-\eta^{2}\right)\right]^{\frac{s+2}{2}}} \\
& \times \int_{k_{\min }}^{k_{\max }} \mathrm{d} k k^{-s} \delta\left[\left(v \mu-j V_{\mathrm{A}}\right) \eta k+n \Omega\right]\left(\frac{n J_{n}(W)}{W}\right)^{2}
\end{aligned}
$$

with

$W=k R_{\mathrm{L}} \sqrt{\left(1-\mu^{2}\right)\left(1-\eta^{2}\right)}$

where $I_{0}^{j}$ reflects the two intensity components of turbulence forward and backward to the ambient magnetic field, and we have taken both to have the same spectral shape to be in accord with observations. Then $I_{0}^{+}+I_{0}^{-}=I_{0}$, where $I_{0}$ is given by Eq. LS(4). In Eq. (2) we introduced the cosmic ray particle gyroradius $R_{\mathrm{L}}=v /|\Omega|$.

One immediately notes that if $n=0$ in Eq. (1) all three Fokker-Planck coefficients are zero, so there is no transit-time damping contribution for Alfvèn waves. The contributions for therefore $n \neq 0$ are customarily referred to as gyroresonant contributions.

Because we are concerned with the transport of very energetic particles $v \gg V_{\mathrm{A}}$ we do not lose much generality if we extend the turbulence power spectrum to infinetely large 
wavenumbers, i.e. $k_{\max }=l_{\min }^{-1}=\infty$. After some straightforward algebra, one can write the gyroresonance contributions as

$$
\begin{aligned}
\left(\begin{array}{c}
D_{\mu \mu} \\
D_{\mu p} \\
D_{p p}
\end{array}\right) & =\frac{4 \pi^{2} \Omega^{2} R_{\mathrm{L}}^{s}}{B_{0}^{2} v} \sum_{j= \pm 1} I_{0}^{j}\left(\begin{array}{c}
(1-j \mu \epsilon)^{2} \\
j \epsilon p(1-j \mu \epsilon) \\
(\epsilon p)^{2}
\end{array}\right)|\mu-j \epsilon|^{s+1} \sum_{n=1}^{n_{\max }} n^{-s} \\
& \times \int_{\eta_{\min }}^{\min \left[1, \eta_{\max }\right]} \mathrm{d} \eta \frac{\left(1+\eta^{2}\right)}{\left(1-\eta^{2}\right)} \frac{\eta^{s+1}}{\left[\eta^{2}+\Lambda\left(1-\eta^{2}\right)\right]^{\frac{s+2}{2}}} \\
& \times J_{n}^{2}\left(n \frac{\sqrt{1-\eta^{2}}}{\eta} \frac{\sqrt{1-\mu^{2}}}{|\mu-j \epsilon|}\right)
\end{aligned}
$$

where $\epsilon=v_{\mathrm{A}} / v \ll 1$. Together with

$\eta_{\min }=\frac{n}{R_{\mathrm{L}} k_{\max }|\mu-j \epsilon|}=0$,

$\eta_{\max }=\frac{n}{R_{\mathrm{L}} k_{\min }|\mu-j \epsilon|}$

and

$n_{\max }=|\mu-\epsilon j| R_{\mathrm{L}} k_{\max }=\infty$

we obtain

$$
\begin{aligned}
\left(\begin{array}{c}
D_{\mu \mu} \\
D_{\mu p} \\
D_{p p}
\end{array}\right) & =\frac{4 \pi^{2} \Omega^{2} R_{\mathrm{L}}^{s}}{B_{0}^{2} v} \sum_{j= \pm 1} I_{0}^{j}\left(\begin{array}{c}
(1-j \mu \epsilon)^{2} \\
j \epsilon p(1-j \mu \epsilon) \\
(\epsilon p)^{2}
\end{array}\right)|\mu-j \epsilon|^{s+1} \sum_{n=1}^{\infty} n^{-s} \\
& \times \int_{0}^{\min \left[1, \eta_{\max }\right]} d \eta \frac{\left(1+\eta^{2}\right)}{\left(1-\eta^{2}\right)} \frac{\eta^{s+1}}{\left[\eta^{2}+\Lambda\left(1-\eta^{2}\right)\right]^{\frac{s+2}{2}}} \\
& \times J_{n}^{2}\left(n \frac{\sqrt{1-\eta^{2}}}{\eta} \frac{\sqrt{1-\mu^{2}}}{|\mu-j \epsilon|}\right) .
\end{aligned}
$$

Without further information on the relative strengths of $I_{0}^{-}$to $I_{0}^{+}$ it is not possible to take the gyroresonance contributions much further. In the following we treat the symmetric case where $I_{0}^{-}=I_{0}^{+}$, to illuminate the changes in the Fokker-Planck coefficients brought about by the anisotropic nature of the plasma wave turbulence.

\section{Anisotropic gyroresonance effects}

\subsection{Rate of adiabatic deceleration}

From Eq. (7) we obtain for the symmetric wave case $I_{0}^{-}=I_{0}^{+}$

$$
\begin{aligned}
D_{\mu p} & =\frac{2 \pi^{2} \Omega^{2} R_{\mathrm{L}}^{s} I_{0}}{B_{0}^{2} v} \sum_{j= \pm 1} j \epsilon p(1-j \mu \epsilon)|\mu-j \epsilon|^{s+1} \sum_{n=1}^{\infty} n^{-s} \\
& \times \int_{0}^{\min \left[1, \eta_{\max }\right]} \mathrm{d} \eta \frac{\left(1+\eta^{2}\right)}{\left(1-\eta^{2}\right)} \frac{\eta^{s+1}}{\left[\eta^{2}+\Lambda\left(1-\eta^{2}\right)\right]^{\frac{s+2}{2}}} \\
& \times J_{n}^{2}\left(n \frac{\sqrt{1-\eta^{2}}}{\eta} \frac{\sqrt{1-\mu^{2}}}{|\mu-j \epsilon|}\right) .
\end{aligned}
$$

Note that $D_{\mu p}(-\mu)=-D_{\mu p}(\mu)$ is antisymmetric in $\mu$, so that the rate of adiabatic acceleration $D$ in Eq. $(\operatorname{LS}(8))$

$D=\frac{3 v}{4 p} \int_{-1}^{1} \mathrm{~d} \mu\left(1-\mu^{2}\right) \frac{D_{\mu p}}{D_{\mu \mu}}=0$

is identically zero in the symmetric wave intensity case $I_{0}^{+}=$ $I_{0}^{-}=I_{0} / 2$.

\subsection{Pitch-angle Fokker-Planck coefficient}

Again from Eq. (7) we obtain

$$
\begin{aligned}
D_{\mu \mu} & =\frac{2 \pi^{2} \Omega^{2} R_{\mathrm{L}}^{s} I_{0}}{B_{0}^{2} v} \sum_{j= \pm 1}(1-j \mu \epsilon)^{2}|\mu-j \epsilon|^{s+1} \sum_{n=1}^{\infty} n^{-s} \\
& \times \int_{0}^{\min \left[1, \eta_{\max }\right]} \mathrm{d} \eta \frac{\left(1+\eta^{2}\right)}{\left(1-\eta^{2}\right)} \frac{\eta^{s+1}}{\left[\eta^{2}+\Lambda\left(1-\eta^{2}\right)\right]^{\frac{s+2}{2}}} \\
& \times J_{n}^{2}\left(n \frac{\sqrt{1-\eta^{2}}}{\eta} \frac{\sqrt{1-\mu^{2}}}{|\mu-j \epsilon|}\right)
\end{aligned}
$$

and

$$
\begin{aligned}
D_{p p} & =\frac{2 \pi^{2} \Omega^{2} R_{\mathrm{L}}^{s} I_{0} \epsilon^{2} p^{2}}{B_{0}^{2} v} \sum_{j= \pm 1}|\mu-j \epsilon|^{s+1} \sum_{n=1}^{\infty} n^{-s} \\
& \times \int_{0}^{\min \left[1, \eta_{\max }\right]} \mathrm{d} \eta \frac{\left(1+\eta^{2}\right)}{\left(1-\eta^{2}\right)} \frac{\eta^{s+1}}{\left[\eta^{2}+\Lambda\left(1-\eta^{2}\right)\right]^{\frac{s+2}{2}}} \\
& \times J_{n}^{2}\left(n \frac{\sqrt{1-\eta^{2}}}{\eta} \frac{\sqrt{1-\mu^{2}}}{|\mu-j \epsilon|}\right) .
\end{aligned}
$$

Now we must approximate the term $|\mu-j \epsilon|$. For doing this we consider two cases.

\subsubsection{Small values $|\mu| \leq \epsilon$}

This case is treated in detail in Appendices A and B, where we derive

$D_{\mu \mu} \simeq \frac{2 \pi^{2} \Omega^{2} R_{\mathrm{L}}^{s} I_{0}}{B_{0}^{2} v} \epsilon^{s+1} W_{s}(\Lambda, M, s)$

with

$W_{s}(\Lambda, M, s) \simeq$

$$
\left\{\begin{array}{ccc}
\frac{M^{2}}{s \Lambda}\left[1+\frac{1-(1+\Lambda)^{\frac{2-s}{2}}}{(2-s) \Lambda}\right] & \text { for } & M^{2} \ll 1, M^{2} \ll \Lambda \\
\frac{1}{4}+\frac{2 \zeta(s+1)}{\pi} & \text { for } & \Lambda \ll M^{2} \ll 1 \\
\frac{M^{2}}{s \Lambda} & \text { for } & 1 \ll M^{2} \ll \Lambda \\
\frac{1}{2}+\frac{4 \zeta(s+1)}{\pi} & \text { for } & M^{2} \gg 1, M^{2} \gg \Lambda .
\end{array}\right.
$$

With

$M=\frac{1}{\epsilon} \gg 1$

Equation (13) reduces to

$W_{s}(\Lambda, \epsilon, s) \simeq\left\{\begin{array}{cc}\frac{1}{s \Lambda \epsilon^{2}} & \text { for } \quad \Lambda \epsilon^{2} \gg 1 \\ \frac{1}{2}+\frac{4 \zeta(s+1)}{\pi} & \text { for } \quad \Lambda \epsilon^{2} \ll 1 .\end{array}\right.$ 


\subsubsection{Large values $|\mu|>\epsilon$}

For large pitch angles $|\mu|>\epsilon$ we obtain (again from Appendices A and B)

$D_{\mu \mu}^{G}=\frac{2 \pi^{2} \Omega^{2} R_{\mathrm{L}}^{s} I_{0}}{B_{0}^{2} v}|\mu|^{s+1} W_{l}(\Lambda, M, s)$

with

$W_{l}(\Lambda, M, s) \simeq$

$$
\left\{\begin{aligned}
\frac{M^{2}}{s \Lambda}\left[1+\frac{1-(1+\Lambda)^{\frac{2-s}{2}}}{(2-s) \Lambda}\right] & \text { for } & M^{2} \ll 1, M^{2} \ll \Lambda \\
\frac{1}{4}+\frac{2 \zeta(s+1)}{\pi} & \text { for } & \Lambda \ll M^{2} \ll 1 \\
\frac{M^{2}}{s \Lambda} & \text { for } & 1 \ll M^{2} \ll \Lambda \\
\frac{1}{2}+\frac{4 \zeta(s+1)}{\pi} & \text { for } & M^{2} \gg 1, M^{2} \gg \Lambda
\end{aligned}\right.
$$

but now with

$M=\frac{\sqrt{1-\mu^{2}}}{\mu}$.

\section{Cosmic ray momentum diffusion from Alfvèn waves}

Using Eq. (10) and the definition of the momentum diffusion coefficient $A$ (Eq. LS(9)) we obtain for cosmic rays with gyroradii much less than $R_{\mathrm{L}} \ll l_{\max } / 2 \pi$

$A=\frac{\pi}{2}(s-1) \frac{(\delta B)^{2}}{B_{0}^{2}}\left(k_{\min } R_{\mathrm{L}}\right)^{s-1} \frac{v \epsilon^{2} p^{2}}{R_{\mathrm{L}}} h(\Lambda, \epsilon, s)$.

In deriving this equation we have used

$I_{0}=\frac{(s-1) k_{\min }^{s-1} \delta B^{2}}{4 \pi J(\Lambda, s)}$,

$D_{p p}=(\epsilon p)^{2} D_{\mu \mu}$

and

$A \simeq \frac{1}{2} \int_{-1}^{+1} \mathrm{~d} \mu D_{p p}(\mu)$

to lowest order in $\epsilon$. For the anisotropy function

$$
\begin{aligned}
h(\Lambda, \epsilon, s)= & \frac{1}{J(\Lambda, s)} \int_{0}^{\epsilon} \mathrm{d} \mu \epsilon^{s+1} W_{s}(\mu) \\
& +\frac{1}{J(\Lambda, s)} \int_{\epsilon}^{1} \mathrm{~d} \mu \mu^{s+1} W_{l}(\mu)
\end{aligned}
$$

and with Eq. (15) we obtain

$h(\Lambda, \epsilon, s) \simeq \frac{W_{s}(\Lambda, \epsilon, s)}{J(\Lambda, s)} \epsilon^{s+2}+\frac{1}{J(\Lambda, s)} \int_{\epsilon}^{1} \mathrm{~d} \mu \mu^{s+1} W_{l}(\mu)$

with the integral

$$
\begin{aligned}
J(\Lambda, s) & =\int_{0}^{1} \mathrm{~d} \eta\left[\eta^{2}+\Lambda\left(1-\eta^{2}\right)\right]^{-(2+s) / 2} \\
& ={ }_{2} F_{1}\left(\frac{s+2}{2}, 1 ; \frac{3}{2} ; 1-\Lambda\right)
\end{aligned}
$$

from LS.

\subsection{Isotropic turbulence $\Lambda=1$}

For this case it is easy to write down $W_{l}$ (see Eq. (17))

$W_{l}=\left\{\begin{array}{ccc}\frac{M^{2}}{s} c_{1} & \text { for } & M^{2} \ll 1 \\ \frac{1}{2}+\frac{4 \zeta(s+1)}{\pi} & \text { for } & M^{2} \gg 1\end{array}\right.$

with

$M^{2}=\frac{1}{\mu^{2}}-1$

and

$c_{1}=1+\frac{1-2^{\frac{2-s}{2}}}{2-s}$

For $h$ we use $J(\Lambda=1, s)=1$ from Eq. (24) and split the second integral of Eq. (23)

$h(\Lambda=1, \epsilon, s) \approx\left(\frac{1}{2}+\frac{4 \zeta(s+1)}{\pi}\right) \epsilon^{s+2}$

$+\left(\frac{1}{2}+\frac{4 \zeta(s+1)}{\pi}\right) \int_{\epsilon}^{1 / \sqrt{2}} \mathrm{~d} \mu \mu^{s+1}+\frac{c_{1}}{s} \int_{1 / \sqrt{2}}^{1} \mathrm{~d} \mu\left(\mu^{s-1}-\mu^{s+1}\right)$.

These integrals are elementary and we obtain to lowest order in $\epsilon$

$$
\begin{aligned}
h(\Lambda=1, s) \approx & \left(\frac{1}{2}+\frac{4 \zeta(s+1)}{\pi}\right) \frac{2^{-\frac{s+2}{2}}}{s+2} \\
& +\frac{c_{1}}{s}\left(\frac{1}{s}-\frac{2^{-s / 2}}{s}+\frac{2^{-\frac{s+2}{2}}}{(s+2)}-\frac{1}{(s+2)}\right) .
\end{aligned}
$$

\subsection{Strongly perpendicular turbulence $\Lambda \ll 1$}

In this case we obtain from Eq. (17)

$W_{l}=\left\{\begin{array}{ccc}\frac{M^{2}}{s \Lambda} f_{1}(\Lambda) & \text { for } & M^{2} \ll \Lambda \ll 1 \\ \frac{1}{4}+\frac{2 \zeta(s+1)}{\pi} & \text { for } & \Lambda \ll M^{2} \ll 1 \\ \frac{1}{2}+\frac{4 \zeta(s+1)}{\pi} & \text { for } & M^{2} \gg 1\end{array}\right.$

with the function

$f_{1}(\Lambda)=1+\frac{1-(1+\Lambda)^{(2-s) / 2}}{(2-s) \Lambda}$.

For $h$ we now find

$$
\begin{aligned}
h(\Lambda \ll 1, s) & \approx\left(\frac{1}{2}+\frac{4 \zeta(s+1)}{\pi}\right) \frac{\epsilon^{s+2}}{J(\Lambda, s)} \\
& +\frac{1}{J(\Lambda, s)}\left(\frac{1}{2}+\frac{4 \zeta(s+1)}{\pi}\right) \int_{\epsilon}^{1 / \sqrt{2}} \mathrm{~d} \mu \mu^{s+1} \\
& +\frac{1}{J(\Lambda, s)}\left(\frac{1}{4}+\frac{2 \zeta(s+1)}{\pi}\right) \int_{1 / \sqrt{2}}^{1 / \sqrt{1+\Lambda}} \mathrm{d} \mu \mu^{s+1} \\
& +\frac{1}{J(\Lambda, s)}\left(\frac{1}{2 s \Lambda}+\frac{1}{8}\right) \int_{1 / \sqrt{1+\Lambda}}^{1} \mathrm{~d} \mu \mu^{s+1}\left(\frac{1}{\mu^{2}}-1\right) .
\end{aligned}
$$


These integrals are elementary too, so that

$$
\begin{aligned}
h(\Lambda \ll 1, s)= & \frac{1}{J(\Lambda, s)}\left(\frac{1}{2}+\frac{4 \zeta(s+1)}{\pi}\right) \frac{1}{(s+2) 2^{(s+2) / 2}} \\
& +\frac{1}{J(\Lambda, s)}\left(\frac{1}{4}+\frac{2 \zeta(s+1)}{\pi}\right) \frac{1}{s+2} \\
& \times\left(\frac{1}{(1+\Lambda)^{(s+2) / 2}}-\frac{1}{2^{(s+2) / 2}}\right) \\
& +\frac{1}{J(\Lambda, s)}\left(\frac{1}{2 s \Lambda}+\frac{1}{8}\right)\left(\frac{1}{s}-\frac{1}{s+2}\right) \\
& -\frac{1}{J(\Lambda, s)}\left(\frac{1}{2 s \Lambda}+\frac{1}{8}\right) \\
& \times\left(\frac{1}{s(1+\Lambda)^{s / 2}}-\frac{1}{(s+2)(1+\Lambda)^{(s+2) / 2}}\right)
\end{aligned}
$$

to lowest order in $\epsilon$. If we use $\Lambda \ll 1$ we find

$$
\begin{aligned}
h(\Lambda \ll 1, s) \approx & \frac{1}{J(\Lambda, s)}\left(\frac{1}{2}+\frac{4 \zeta(s+1)}{\pi}\right) \\
& \times \frac{1}{2(s+2)}\left(1+\frac{1}{2^{(s+2) / 2}}\right) .
\end{aligned}
$$

Now we must calculate $J(\Lambda \ll 1, s)$. To do this we can use

$$
\begin{aligned}
J(\Lambda, s) & ={ }_{2} F_{1}\left(\frac{s+2}{2}, 1, \frac{3}{2}, 1-\Lambda\right) \\
& =\frac{1}{\Lambda^{\frac{s+1}{2}}}{ }_{2} F_{1}\left(\frac{1-s}{2}, \frac{1}{2}, \frac{3}{2}, 1-\Lambda\right)
\end{aligned}
$$

to obtain

$$
J(\Lambda \ll 1, s) \approx \frac{1}{\Lambda^{\frac{s+1}{2}}}{ }_{2} F_{1}\left(\frac{1-s}{2}, \frac{1}{2}, \frac{3}{2}, 1\right)=\frac{\sqrt{\pi}}{2 \Lambda^{\frac{s+1}{2}}} \frac{\Gamma\left(\frac{s+1}{2}\right)}{\Gamma\left(\frac{s+2}{2}\right)} .
$$

According to Eq. (34) we thus derive

$$
\begin{aligned}
h(\Lambda \ll 1, s) \approx & \frac{2 \Lambda^{(s+1) / 2}}{\sqrt{\pi}} \frac{\Gamma\left(\frac{s+2}{2}\right)}{\Gamma\left(\frac{s+1}{2}\right)}\left(\frac{1}{2}+\frac{4 \zeta(s+1)}{\pi}\right) \\
& \times \frac{1}{2(s+2)}\left(1+\frac{1}{2^{(s+2) / 2}}\right) .
\end{aligned}
$$

\subsection{Strongly parallel turbulence $\epsilon^{-2} \gg \Lambda \gg 1$}

In this case we obtain

$$
W_{l}=\left\{\begin{array}{ccc}
\frac{M^{2}}{\Lambda s} f_{1}(\Lambda) & \text { for } & M^{2} \ll 1 \\
\frac{M^{2}}{\Lambda s} & \text { for } & 1 \ll M^{2} \ll \Lambda \\
\frac{1}{2}+\frac{4 \zeta(s+1)}{\pi} & \text { for } & M^{2} \gg \Lambda \gg 1
\end{array}\right.
$$

and for $h$ we now find

$$
\begin{aligned}
h\left(\epsilon^{-2} \gg \Lambda \gg 1, s\right) \approx\left(\frac{1}{2}+\frac{4 \zeta(s+1)}{\pi}\right) \frac{\epsilon^{s+2}}{J(\Lambda, s)} \\
+\frac{1}{J(\Lambda, s)}\left(\frac{1}{2}+\frac{4 \zeta(s+1)}{\pi}\right) \int_{\epsilon}^{1 / \sqrt{1+\Lambda}} \mathrm{d} \mu \mu^{s+1} \\
+\frac{1}{J(\Lambda, s) s \Lambda} \int_{1 / \sqrt{1+\Lambda}}^{1} \mathrm{~d} \mu \mu^{s+1}\left(\frac{1}{\mu^{2}}-1\right)
\end{aligned}
$$

Table 1. Anisotropy function $h$ for different anisotropy parameters.

\begin{tabular}{lll}
\hline \hline Anisotropy parameter $\Lambda$ & $h(\Lambda, s=5 / 3)$ & $h_{\text {num }}(\Lambda, s=5 / 3)$ \\
\hline$\Lambda=1$ & 0.204 & 0.077 \\
$\Lambda \ll 1$ & $0.443 \cdot \Lambda^{\frac{s+1}{2}}$ & $0.470 \cdot \Lambda^{\frac{s+1}{2}}$ \\
$\Lambda \gg 1$ & 0.327 & 0.327 \\
\hline
\end{tabular}

where we have used

$f_{1}(\Lambda \gg 1) \approx 1$

Like in the cases before the integrals are elementary yielding

$h\left(\epsilon^{-2} \gg \Lambda \gg 1, s\right) \approx \frac{1}{J(\Lambda, s) s \Lambda}\left(\frac{1}{s}-\frac{1}{s+2}\right)$.

Now we must calculate $J(\Lambda \gg 1, s)$. With Eq. (24) it is easy to show that

$$
\begin{aligned}
J(\Lambda \gg 1, s) & ={ }_{2} F_{1}\left(\frac{s+2}{2}, 1 ; \frac{3}{2} ; 1-\Lambda\right) \\
& \approx \frac{1}{\Lambda}{ }_{2} F_{1}\left(\frac{1-s}{2}, 1 ; \frac{3}{2} ; 1\right)=\frac{1}{s \Lambda} .
\end{aligned}
$$

So the final result for this case is

$h\left(\epsilon^{-2} \gg \Lambda \gg 1, s\right)=\frac{1}{s}-\frac{1}{s+2}=\frac{2}{s(s+2)}$.

\subsection{Extremely parallel turbulence $\Lambda \gg \epsilon^{-2} \gg 1$}

In this case we always have

$W_{l}=\frac{M^{2}}{\Lambda s}$

and

$W_{s}=\frac{\epsilon^{-2}}{s \Lambda}$

and with Eq. (42) we obtain for the function $h$

$h\left(\Lambda \gg \epsilon^{-2} \gg 1, s\right) \approx \epsilon^{s}+\int_{\epsilon}^{1} \mathrm{~d} \mu \mu^{s+1} M^{2}$.

To lowest order in $\epsilon$ we find the same result as in the third case

$h\left(\Lambda \gg \epsilon^{-2} \gg 1, s\right)=\frac{1}{s}-\frac{1}{s+2}=\frac{2}{s(s+2)}$.

So we always have in the case of parallel turbulence

$h(\Lambda \gg 1, s)=\frac{2}{s(s+2)}$.

In Table 1 we have listed the results for the three different cases of anisotropy and for $s=5 / 3$. Also we have calculated the three cases numerically. The agreement ist very good in the cases two and three and acceptable in the first case. Figure 1 shows the numerical result for the case $s=5 / 3$. 


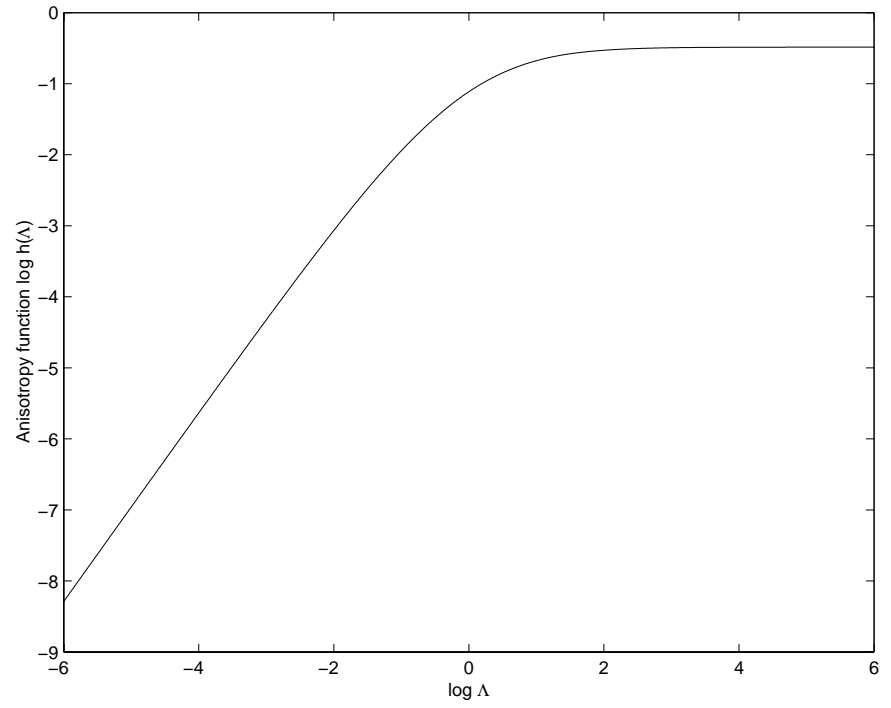

Fig. 1. Numerical result for the anisotropy function $h(\Lambda)$ for the case $s=5 / 3$.

\subsection{Rigidity dependence of the momentum diffusion coefficient}

So the final result of this chapter is, that we can write down the momentum diffusion coefficient $A$ as

$A=\frac{\pi}{2}(s-1) \frac{(\delta B)^{2}}{B_{0}^{2}}\left(k_{\min } R_{\mathrm{L}}\right)^{s-1} \frac{v \epsilon^{2} p^{2}}{R_{\mathrm{L}}} h(\Lambda, s)$

with the energy independent anisotropy function $h(\Lambda, s)$. Introducing the rigidity $r$ and the parameter $r_{0}$

$r=\frac{p c}{|q|}$

$r_{0}=\frac{m c^{2}}{|q|}$

we can write Eq. (49) as

$\frac{A}{A_{0}}=\sqrt{\left(\frac{r_{0}}{M V}\right)^{2}+\left(\frac{r}{M V}\right)^{2}} \cdot\left(\frac{r}{M V}\right)^{s-1} \cdot h(\Lambda, s)$

with

$A_{0}=\frac{\pi}{2}(s-1) \frac{k_{\min }^{s-1}}{B_{0}^{s} c}\left(q \frac{v_{\mathrm{A}} \delta B}{c}\right)^{2}(M V)^{s}$

where we have used $R_{\mathrm{L}}=r / B_{0}$ and $\epsilon=v_{\mathrm{A}} / c \cdot \sqrt{r_{0}^{2}+r^{2}} / r$. It is easy to see, that we obtain the same $A_{0}$ for electrons, positrons and protons. Figure 2 shows $A / A_{0}$ as a function of the rigidity for electrons, positrons and protons for small, medium and large values of the anisotropy parameter $\Lambda$ and $s=5 / 3$. Figure 1 shows the rigidity independent function $h(\Lambda, s=5 / 3)$. At nonrelativistic rigidities we find $A \propto r^{s-1}$ whereas at relativistic rigidities $A \propto r^{s}$. Also note that for relativistic rigidities electrons, positrons and protons approach the same $A / A_{0}$. For all the calculations we restricted our analysis to $1<s<2$, $R_{\mathrm{L}} k_{\min } \ll 1$ and $\epsilon \ll 1$. Note also that for electrons and positrons of rigidities $r<10^{2} M V$ deviations of the Alfvèn branch dispersion relation from the linear form $\omega= \pm v_{\mathrm{A}} k_{\|}$have been neglected in our calculations.

\section{Cosmic ray spatial diffusion from Alfvèn waves}

From LS and with $D_{\mu \mu}(\mu)=D_{\mu \mu}(-\mu)$ we have

$\kappa=\frac{v^{2}}{4} \int_{0}^{1} \mathrm{~d} \mu \frac{\left(1-\mu^{2}\right)^{2}}{D_{\mu \mu}(\mu)}$.

Together with Eq. (12) and Eq. (16) we obtain

$\kappa=\frac{v B_{0}^{2} R_{\mathrm{L}}^{2-s}}{2 \pi(s-1) \delta B^{2} k_{\min }^{s-1}} g(s, \epsilon, \Lambda)$

with

$g(s, \epsilon, \Lambda)=\frac{J(\Lambda, s)}{\epsilon^{s+1}} \int_{0}^{\epsilon} \mathrm{d} \mu \frac{1}{W_{s}(\mu)}+J(\Lambda, s) \int_{\epsilon}^{1} \mathrm{~d} \mu \frac{\left(1-\mu^{2}\right)^{2}}{\mu^{s+1} W_{l}(\mu)}$

and

$W_{s}(s, \epsilon, \Lambda)=\left\{\begin{array}{ccc}\frac{1}{s \Lambda \epsilon^{2}} & \text { for } & \Lambda \epsilon^{2} \gg 1 \\ \frac{1}{2}+\frac{4 \zeta(s+1)}{\pi} & \text { for } & \Lambda \epsilon^{2} \ll 1\end{array}\right.$

and with Eq. (17) for $W_{l}$. This can be written as

$g(s, \epsilon, \Lambda) \approx \frac{J(\Lambda, s)}{W_{s}(s, \epsilon, \Lambda)} \epsilon^{-s}+J(\Lambda, s) \int_{\epsilon}^{1} \mathrm{~d} \mu \frac{\left(1-\mu^{2}\right)^{2}}{\mu^{s+1} W_{l}(\mu)}$.

\subsection{Isotropic turbulence $\Lambda=1$}

Here the function $\mathrm{g}$ can be written as

$g(s, \epsilon, \Lambda) \approx\left(\frac{1}{2}+\frac{4 \zeta(s+1)}{\pi}\right)^{-1} \epsilon^{-s}+\int_{\epsilon}^{1} \mathrm{~d} \mu \frac{\left(1-\mu^{2}\right)^{2}}{\mu^{s+1} W_{l}(\mu)}$

what is equal to

$$
\begin{aligned}
g(s, \epsilon, \Lambda) \approx & \left(\frac{1}{2}+\frac{4 \zeta(s+1)}{\pi}\right)^{-1} \epsilon^{-s} \\
& +\left(\frac{1}{2}+\frac{4 \zeta(s+1)}{\pi}\right)^{-1} \int_{\epsilon}^{1 / \sqrt{2}} \mathrm{~d} \mu \frac{\left(1-\mu^{2}\right)^{2}}{\mu^{s+1}} \\
& +\frac{s}{c_{1}} \int_{1 / \sqrt{2}}^{1} \mathrm{~d} \mu \frac{\left(1-\mu^{2}\right)^{2}}{\mu^{s+1} M^{2}} .
\end{aligned}
$$

To lowest order in $\epsilon$ we finally find

$g(s, \epsilon, \Lambda=1) \approx\left(\frac{1}{2}+\frac{4 \zeta(s+1)}{\pi}\right)^{-1}\left(1+\frac{1}{s}\right) \epsilon^{-s}$.

\subsection{Strongly perpendicular turbulence $\Lambda \ll 1$}

For this case we can do the same calculations and approximations as in the case before and we obtain

$g(s, \Lambda \ll 1) \approx J(\Lambda \ll 1, s)\left(\frac{1}{2}+\frac{4 \zeta(s+1)}{\pi}\right)^{-1}\left(1+\frac{1}{s}\right) \epsilon^{-s}$

\subsection{Strongly parallel turbulence $\epsilon^{-2} \gg \Lambda \gg 1$}

But in the third case we obtain with our approximations

$$
\begin{aligned}
g\left(s, \epsilon^{-2} \gg \Lambda \gg 1\right) \approx & J(\Lambda \gg 1, s)\left(\frac{1}{2}+\frac{4 \zeta(s+1)}{\pi}\right)^{-1}\left(1+\frac{1}{s}\right) \epsilon^{-s} \\
& +J(\Lambda \gg 1, s) s \Lambda\left(\frac{1}{2-s}-\frac{1}{4-s}\right)
\end{aligned}
$$




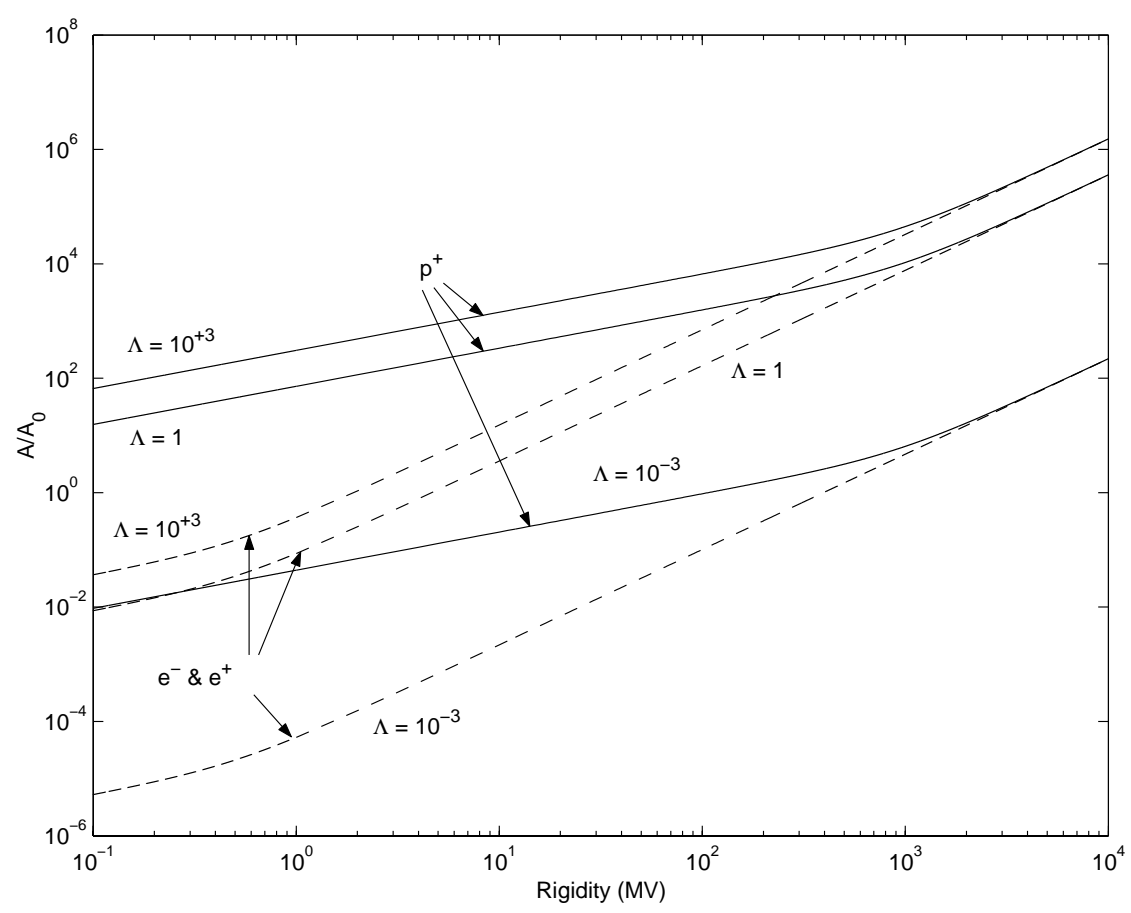

Fig. 2. The momentum diffusion coefficient $A / A_{0}$ for electrons, positrons and protons for $\Lambda=0.001,1,1000$.

\subsection{Extremely parallel turbulence $\Lambda \gg \epsilon^{-2} \gg 1$}

In this last case we obtain for the function $\mathrm{g}$

$g\left(s, \Lambda \gg \epsilon^{-2} \gg 1\right) \approx \epsilon^{2}+\int_{\epsilon}^{1} \mathrm{~d} \mu \frac{\left(1-\mu^{2}\right)^{2}}{\mu^{s+1} M^{2}}$

where we used Eq. (42) again. To lowest order in $\epsilon$ we finally obtain

$g\left(s, \Lambda \gg \epsilon^{-2} \gg 1\right) \approx\left(\frac{1}{2-s}-\frac{1}{4-s}\right)=\frac{2}{(2-s)(4-s)}$.

If we restrict our analysis to the case of $\Lambda \epsilon^{s} \ll 1$ we always have

$g\left(s, \Lambda \epsilon^{s} \ll 1\right) \approx J(\Lambda, s)\left(\frac{1}{2}+\frac{4 \zeta(s+1)}{\pi}\right)^{-1}\left(1+\frac{1}{s}\right) \epsilon^{-s}$.

We have also calculated the ratio $g / J$ for the this case and for $s=5 / 3$ numerically. For $s=5 / 3$ the analytic result is

$\frac{g\left(s, \Lambda \epsilon^{s} \ll 1\right)}{J(\Lambda, s)} \approx 0.749 \epsilon^{-s}$

whereas the numerical calculations yields

$\frac{g\left(s, \Lambda \epsilon^{s} \ll 1\right)}{J(\Lambda, s)} \approx 0.623 \epsilon^{-s}$

in reasonable agreement. For $s=5 / 3$ we calculated the function $g$ for different anisotropy parameters in Table 2 .
Table 2. Anisotropy function $g$ for different anisotropy parameters.

\begin{tabular}{ll}
\hline \hline Anisotropy parameter $\Lambda$ & $g(\Lambda, s=5 / 3)$ \\
\hline$\Lambda \ll 1$ & $0.63 \Lambda^{-(s+1) / 2} \epsilon^{-s}$ \\
$\Lambda=1$ & $0.75 \epsilon^{-s}$ \\
$\epsilon^{-s} \gg \Lambda \gg 1$ & $0.45 \Lambda^{-1} \epsilon^{-s}$ \\
\hline
\end{tabular}

\subsection{Rigidity dependence of the spatial diffusion coefficient}

If we restrict our analysis to $\Lambda \epsilon^{s} \ll 1$ we obtain for the spatial diffusion coefficient

$\frac{\kappa}{\kappa_{0}}=\frac{\left(\frac{r}{M V}\right)^{3}}{\left[\left(\frac{r_{0}}{M V}\right)^{2}+\left(\frac{r}{M V}\right)^{2}\right]^{(s+1) / 2}} \cdot J(\Lambda, s)$

with

$\kappa_{0}=\frac{1}{\pi+8 \zeta(s+1)} \frac{s+1}{s(s-1)} \frac{c k_{\min }}{\delta B^{2}}\left(\frac{c B_{0}}{v_{\mathrm{A}} k_{\min }}\right)^{s}(M V)^{2-s}$.

It is easy to see, that we obtain the same $\kappa_{0}$ for electrons, positrons and protons. Figure 3 shows the function $J(\Lambda, s=$ $5 / 3$ ) and Fig. 4 shows $\kappa / \kappa_{0}$ as a function of the rigidity for electrons, positrons and protons for small, medium and large values of the anisotropy parameter $\Lambda$ and $s=5 / 3$. For nonrelativistic rigidities we obtain $R_{\mathrm{L}} \propto r, \epsilon \propto r^{-1}$ and $\kappa \propto R_{\mathrm{L}}^{2-s} \epsilon^{-s-1}$. Therefore we find that for nonrelativistic rigidities the spatial diffusion coefficients are proportional to $r^{3}$ whereas for relativistic rigidities $\kappa \propto r^{2-s}$. At relativistic rigidities the diffusion coefficients of electrons, positrons and protons are the same. 


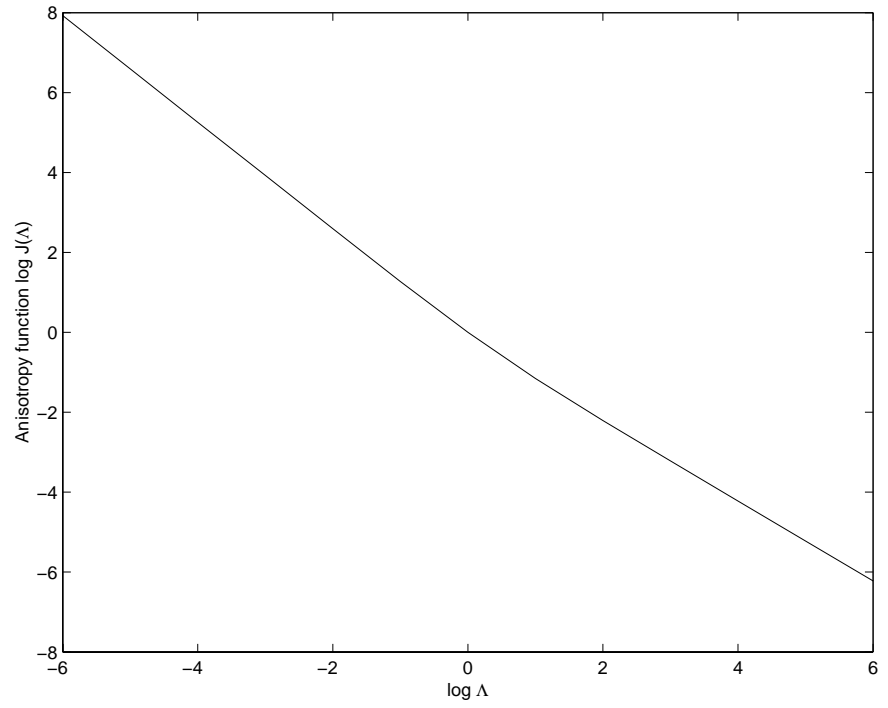

Fig. 3. The anisotropy function $J(\Lambda)$ for the case $s=5 / 3$.

\subsection{The mean free path for Alfvèn turbulence}

We now are able to calculate the mean free path which is connected with the spatial diffusion coefficient through

$\lambda=\frac{3}{v} \kappa$.

This can be written as

$\frac{\lambda}{\lambda_{0}}=\frac{\left(\frac{r}{M V}\right)^{2}}{\left[\left(\frac{r_{0}}{M V}\right)^{2}+\left(\frac{r}{M V}\right)^{2}\right]^{s / 2}} \cdot J(\Lambda, s)$

with

$\lambda_{0}=\frac{3 \kappa_{0}}{c}$.

Figure 5 shows $\lambda / \lambda_{0}$ as a function of the rigidity for electrons, positrons and protons for small, medium and large values of the anisotropy parameter $\Lambda$ and $s=5 / 3$.

We are also able to give asymptotic formulas for the rigidity.

$\frac{\lambda}{\lambda_{0}}\left(r \gg r_{0}\right) \approx r^{2-s} J(\Lambda, s)$,

$\frac{\lambda}{\lambda_{0}}\left(r \ll r_{0}\right) \approx r^{2} \frac{J(\Lambda, s)}{r_{0}^{s}}$.

We can use this formulas for the mean free path if $R_{\mathrm{L}} k_{\min } \leq 1$ and $\Lambda \epsilon^{s} \ll 1$.

\section{Comparison with slab-type Alfvènic turbulence}

In order to discuss the influence of the turbulence anisotropy it is instructive to compare our results with the spatial and momentum diffusion coefficients calculated for pure slab-type turbulence (Schlickeiser 1989, 2002; Dung \& Schlickeiser 1990a,b). In the limit of vanishing magnetic and cross helicity, and turbulence spectral indices $s<2$, one finds

$\kappa_{\mathrm{S}}=\frac{1}{\pi(s-1)(2-s)(4-s)}\left(\frac{B_{0}}{\delta B}\right)^{2} v R_{\mathrm{L}}\left(R_{\mathrm{L}} k_{\min }\right)^{1-s}$ and

$$
A_{\mathrm{S}}=\frac{\pi(s-1)}{s(s+2)}\left(\frac{\delta B}{B_{0}}\right)^{2}\left(R_{\mathrm{L}} k_{\min }\right)^{s-1} \frac{v \epsilon^{2} p^{2}}{R_{\mathrm{L}}} .
$$

\subsection{Momentum diffusion}

Using Eq. (50) we obtain for the ratio of the momentum diffusion coefficient for anisotropic Alfvènic turbulence to slab Alfvènic turbulence

$R_{\mathrm{A}} \equiv \frac{A}{A_{\mathrm{S}}}=\frac{s(s+2)}{2} h(\Lambda, s)$

which is independent of cosmic ray rigidity and only determined by the anisotropy function $h(\Lambda, s)$ and the turbulence spectral index $s$. According to the behaviour of the anisotropy function summarized in Table 1 we find values for the ratio (76) close to unity for isotropic turbulence $(\Lambda=1)$ and strongly parallel turbulence $(\Lambda \gg 1)$, whereas for strongly perpendicular turbulence $(\Lambda \ll 1)$ momentum diffusion is much reduced as compared to the slab case because the ratio (76) varies proportional to $\propto \Lambda^{(s+1) / 2}$. For isotropic and strongly parallel turbulence the momentum diffusion coefficient agree well with the slab momentum diffusion coefficient, both in rigidity dependence and in absolute value.

\subsection{Spatial diffusion}

Using Eq. (60) we obtain for the ratio of the spatial diffusion coefficient for anisotropic turbulence to slab turbulence

$R_{\kappa} \equiv \frac{\kappa}{\kappa_{\mathrm{S}}}=\frac{(2-s)(4-s)}{2} g(\Lambda, s)$

with the anisotropy function $g(\Lambda, s)$ from Table 2 . We first note that for relativistic cosmic ray particles this ratio is independent of cosmic ray rigidity but much larger unity due to the large factor $\epsilon^{-s} \simeq\left(c / V_{\mathrm{A}}\right)^{s}$. Obviously, for non-slab turbulence relativistic cosmic ray diffusion is much more rapid than for slab turbulence, i.e. the diffusion time scale to $\tau_{\mathrm{D}}=L^{2} / \kappa$, where $L$ denotes a length scale, is much shorter in anisotropically distributed Alfvènic turbulence than in slab Alfvènic turbulence of equal total magnitude $\delta B$.

Secondly, we note that for non-relativistic cosmic ray particles the spatial diffusion coefficient ratio (77) becomes smaller due to its rigidity dependence, $R_{K} \propto v^{s} \propto r^{s}$. This modified rigidity dependence can have significant implications for quantitative studies of semirelativistic cosmic rays as the BoronCarbon ratio (e.g. Strong \& Moskalenko 1998; Maurin et al. 2001) that sofar are based on slab transport parameters only.

\section{Summary and conclusions}

With this second paper we have continued to evaluate the relevant cosmic ray transport parameters in the presence of anisotropic plasma wave turbulence. Using the estimates of the anisotropy parameter in the strongly parallel and perpendicular regimes, based on linear Landau damping balancing radiative loss in the diffuse interstellar medium, we have calculated the 


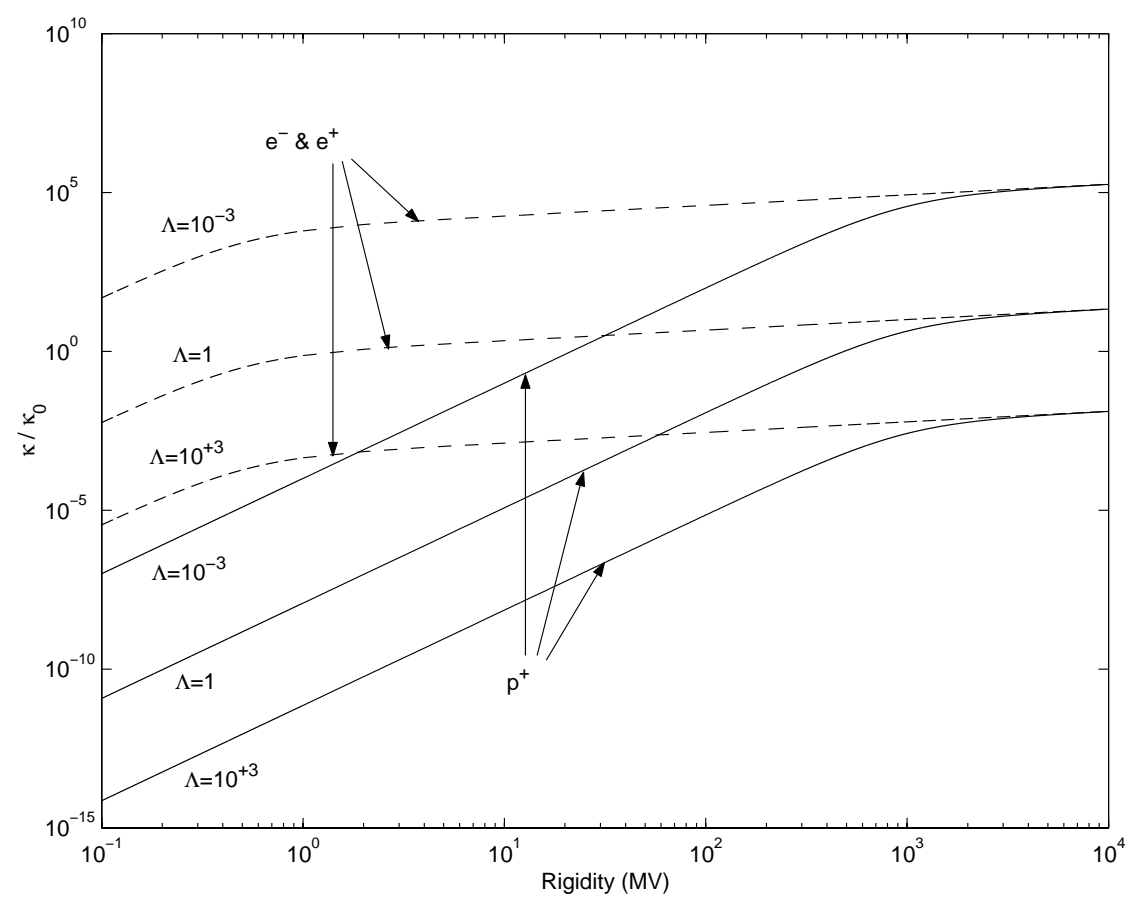

Fig. 4. The spatial diffusion coefficient $\kappa / \kappa_{0}$ for electrons, positrons and protons for $\Lambda=0.001,1,1000$.

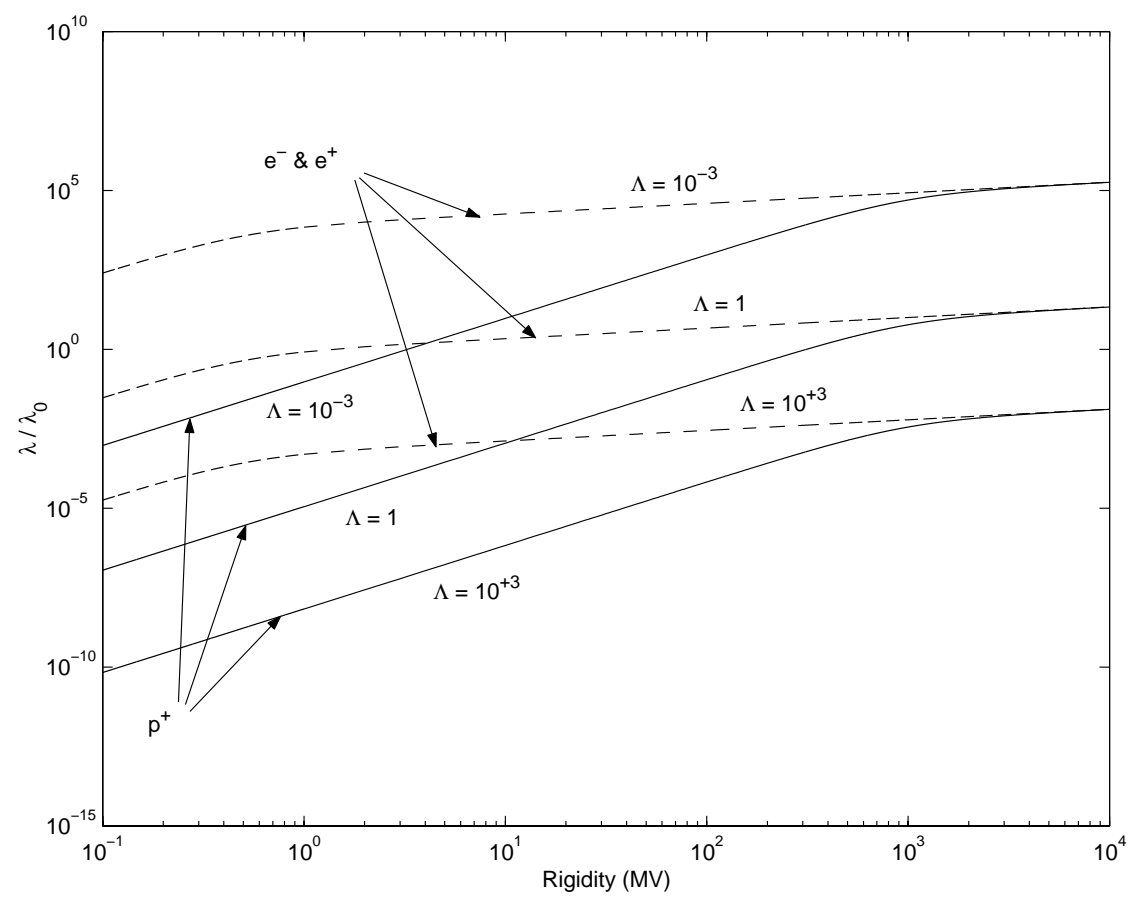

Fig. 5. The mean free path $\lambda / \lambda_{0}$ for electrons, positrons and protons for $\Lambda=0.001,1,1000$.

Fokker-Planck coefficients, the momentum $(A)$ and spatial diffusion coefficient $(\kappa)$ and the mean free path of cosmic ray particles for pure Alfvèn waves. We show that for Alfvèn waves there is no transit-time damping interaction so that the contributions to spatial and momentum diffusion solely result from gyroresonant interactions.

We determined the detailed anisotropy dependence of the spatial and momentum diffusion coefficients in the limits of strongly parallel, strongly perpendicular and isotropic Alfvènic turbulence. We also compared our results with earlier calculations of these two transport parameters in slab Alfvènic turbulence. Here we found that for isotropic and strongly parallel turbulence the momentum diffusion coefficient agree well with the slab momentum diffusion coefficient, both in rigidity dependence and in absolute value. However, in case of spatial diffusion we established that in non-slab Alfvènic turbulence relativistic cosmic ray diffusion is much more rapid than in slab Alfvènic turbulence of equal total magnitude $\delta B$. At non-relativistic cosmic ray rigidities anisotropic Alfvènic 
turbulence yields a different rigidity dependence than slab Alfvènic turbulence.

Combined with results for fast magnetosonic waves (LS) we will be able to determine the cosmic ray transport parameters also for a mixed turbulence, because interstellar plasma turbulence is a mixture of fast magnetosonic waves and shear Alfvèn waves. This analysis will be the subject of the third paper of this series.

Acknowledgements. We gratefully acknowledge support by the Deutsche Forschungsgemeinschaft through Sonderforschungsbereich 191. AT acknowledges the financial support by the Verbundforschung, grant DESY CH1PCA6.

\section{Appendix A: Analysis of the gyroresonant contributions}

We start from Eq. (10)

$$
\begin{aligned}
D_{\mu \mu} & =\frac{2 \pi^{2} \Omega^{2} R_{\mathrm{L}}^{s} I_{0}}{B_{0}^{2} v} \sum_{j= \pm 1}(1-j \mu \epsilon)^{2}|\mu-j \epsilon|^{s+1} \sum_{n=1}^{\infty} n^{-s} \\
& \times \int_{0}^{\min \left[1, \eta_{\max }\right]} \mathrm{d} \eta \frac{\left(1+\eta^{2}\right)}{\left(1-\eta^{2}\right)} \frac{\eta^{s+1}}{\left[\eta^{2}+\Lambda\left(1-\eta^{2}\right)\right]^{\frac{s+2}{2}}} \\
& \times J_{n}^{2}\left(n \frac{\sqrt{1-\eta^{2}}}{\eta} \frac{\sqrt{1-\eta^{2}}}{|\mu-j \epsilon|}\right)
\end{aligned}
$$

and use the substitution $x=\left(1-\eta^{2}\right) / \eta^{2}$. With this transformation we obtain

$$
\begin{aligned}
D_{\mu \mu} & =\frac{\pi^{2} \Omega^{2} R_{\mathrm{L}}^{s} I_{0}}{B_{0}^{2} v} \sum_{j= \pm 1}(1-j \mu \epsilon)^{2}|\mu-j \epsilon|^{s+1} \sum_{n=1}^{\infty} n^{-s} \\
& \times \int_{x_{\min }}^{\infty} \frac{\mathrm{d} x}{x} \frac{x+2}{x+1} \frac{1}{[1+\Lambda x]^{\frac{s+2}{2}}} J_{n}^{2}\left(n \sqrt{x} \frac{\sqrt{1-\mu^{2}}}{|\mu-j \epsilon|}\right)
\end{aligned}
$$

with

$x_{\min }=\frac{1}{\min ^{2}\left[1, \eta_{\max }\right]}-1$.

\section{A.1. Small values $|\mu| \leq \epsilon$}

For small pitch angles $|\mu| \leq \epsilon$ we can use the approximation $|\mu-j \epsilon| \approx \epsilon$ and $\sqrt{1-\mu^{2}} \approx 1$. So we obtain

$$
\begin{aligned}
D_{\mu \mu}= & \frac{\pi^{2} \Omega^{2} R_{\mathrm{L}}^{s} I_{0}}{B_{0}^{2} v} \sum_{j= \pm 1}(1-j \mu \epsilon)^{2} \epsilon^{s+1} \sum_{n=1}^{\infty} n^{-s} \\
& \times \int_{x_{\min }}^{\infty} \frac{\mathrm{d} x}{x} \frac{x+2}{x+1} \frac{J_{n}^{2}(n \sqrt{x} M)}{[1+\Lambda x]^{\frac{s+2}{2}}}
\end{aligned}
$$

with $M=1 / \epsilon$. In lowest order of $\epsilon$ we can use

$$
\sum_{j= \pm 1}(1-j \mu \epsilon)^{2} \approx 2
$$

to obtain approximately

$$
D_{\mu \mu} \simeq \frac{2 \pi^{2} \Omega^{2} R_{\mathrm{L}}^{s} I_{0}}{B_{0}^{2} v} \epsilon^{s+1} \sum_{n=1}^{\infty} n^{-s} \int_{x_{\min }}^{\infty} \frac{\mathrm{d} x}{x} \frac{x+2}{x+1} \frac{J_{n}^{2}(n \sqrt{x} M)}{[1+\Lambda x]^{\frac{s+2}{2}}}
$$

and

$$
D_{p p} \simeq \epsilon^{2} p^{2} D_{\mu \mu}
$$

\section{A.2. Large values $|\mu| \geq \epsilon$}

For pitch angles $|\mu|>\epsilon$ we can use the approximation $|\mu-j \epsilon| \approx|\mu|$ and we obtain

$$
\begin{aligned}
D_{\mu \mu} \simeq & \frac{\pi^{2} \Omega^{2} R_{\mathrm{L}}^{s} I_{0}}{B_{0}^{2} v} \sum_{j= \pm 1}(1-j \mu \epsilon)^{2}|\mu|^{s+1} \sum_{n=1}^{\infty} n^{-s} \\
& \times \int_{x_{\min }}^{\infty} \frac{\mathrm{d} x}{x} \frac{x+2}{x+1} \frac{J_{n}^{2}(n \sqrt{x} M)}{[1+\Lambda x]^{\frac{s+2}{2}}}
\end{aligned}
$$

with

$M=\frac{\sqrt{1-\mu^{2}}}{\mu}$.

To lowest order of $\epsilon$ we can use Eq. (82) again. So we obtain

$$
\begin{aligned}
D_{\mu \mu}^{G} \simeq & \frac{2 \pi^{2} \Omega^{2} R_{\mathrm{L}}^{s} I_{0}}{B_{0}^{2} v}|\mu|^{s+1} \sum_{n=1}^{\infty} n^{-s} \\
& \times \int_{x_{\min }}^{\infty} \frac{\mathrm{d} x}{x} \frac{x+2}{x+1} \frac{J_{n}^{2}(n \sqrt{x} M)}{[1+\Lambda x]^{\frac{s+2}{2}}}, \quad D_{p p} \simeq \epsilon^{2} p^{2} D_{\mu \mu} .
\end{aligned}
$$

For both cases we must calculate the expression

$$
W\left(\Lambda, M, s, x_{\min }\right)=\sum_{n=1}^{\infty} n^{-s} \int_{x_{\min }}^{\infty} \frac{\mathrm{d} x}{x} \frac{x+2}{x+1} \frac{J_{n}^{2}(n \sqrt{x} M)}{[1+\Lambda x]^{\frac{s+2}{2}}}
$$

that is evaluated in Appendix B.

\section{Appendix B: Calculation of expression$$
W\left(\Lambda, M, s, x_{\min }\right)
$$

We restrict the analysis to cosmic ray particles with gyroradii $R_{\mathrm{L}}<k_{\min }^{-1}=l_{\max } / 2 \pi$. In this case $x_{\min }=0$ and we obtain

$$
\begin{aligned}
W\left(\Lambda, M, s, x_{\min }=0\right)= & W(\Lambda, M, s)=\sum_{n=1}^{\infty} n^{-s} \\
& \times \int_{0}^{\infty} \frac{\mathrm{d} x}{x} \frac{x+2}{x+1} \frac{J_{n}^{2}\left(n M x^{1 / 2}\right)}{[1+\Lambda x]^{\frac{s+2}{2}}} .
\end{aligned}
$$

We can split this integral into two parts

$$
\begin{aligned}
& W(\Lambda, M, s)=\int_{0}^{M^{-2}} \frac{\mathrm{d} x}{x} \frac{x+2}{x+1}[1+\Lambda x]^{-(s+2) / 2} \\
& \times \sum_{n=1}^{\infty} n^{-s} J_{n}^{2}\left(n M x^{1 / 2}\right) \\
&+\int_{M^{-2}}^{\infty} \frac{\mathrm{d} x}{x} \frac{x+2}{x+1}[1+\Lambda x]^{-(s+2) / 2} \sum_{n=1}^{\infty} n^{-s} J_{n}^{2}\left(n M x^{1 / 2}\right)
\end{aligned}
$$

and we must appoximate the two sums. To approximate the first sum we use the Kapteyn series (Watson 1966, p. 557) for real $|z| \leq 1$

$\sum_{n=1}^{\infty} \frac{J_{n}^{2}(n z)}{n^{2}}=\frac{z^{2}}{4}$ 
so that

$\sum_{n=1}^{\infty} n^{-s} J_{n}^{2}\left(n M x^{1 / 2}\right) \simeq \sum_{n=1}^{\infty} n^{-2} J_{n}^{2}\left(n M x^{1 / 2}\right)=\frac{M^{2} x}{4}$.

The second sum can be approximated with

$\sum_{n=1}^{\infty} n^{-s} J_{n}^{2}\left(n M x^{1 / 2}\right) \simeq \sum_{n=1}^{\infty} n^{-s} \frac{1}{\pi n M \sqrt{x}}=\frac{\zeta(s+1)}{\pi M \sqrt{x}}$

where we neglect the oscillating part in the approximation of the Bessel function for large arguments (Abramowitz \& Stegun 1972). With Eqs. (92) and (93) we obtain

$W(\Lambda, M, s) \simeq \frac{M^{2}}{4} K_{1}+\frac{\zeta(s+1)}{\pi M} K_{2}$

with the integrals

$K_{1}(M)=\int_{0}^{M^{-2}} \mathrm{~d} x \frac{x+2}{x+1}[1+\Lambda x]^{-\frac{s+2}{2}}$

and

$K_{2}(M)=\int_{M^{-2}}^{\infty} \mathrm{d} x \frac{x+2}{x+1} x^{-3 / 2}[1+\Lambda x]^{-\frac{s+2}{2}}$.

We consider the two integrals in the limits of small and large $M^{2}$ and $\Lambda M^{-2}$ in turn.

\section{B.1. The case of $M^{2} \ll 1$ and $M^{2} \ll \Lambda$}

Here $M^{-2} \gg 1$ so that

$$
\begin{aligned}
K_{1} & \simeq 2 \int_{0}^{M^{-2}} \mathrm{~d} x[1+\Lambda x]^{-\frac{s+2}{2}} \\
& -\int_{0}^{1} \mathrm{~d} x \frac{x}{[1+\Lambda x]^{\frac{s+2}{2}}}-\int_{1}^{M^{-2}} \mathrm{~d} x[1+\Lambda x]^{-\frac{s+2}{2}} \\
& =2 \int_{0}^{M^{-2}} \mathrm{~d} x[1+\Lambda x]^{-\frac{s+2}{2}}-\int_{1}^{M^{-2}} \mathrm{~d} x[1+\Lambda x]^{-\frac{s+2}{2}} \\
& +\frac{2}{s} \frac{\partial}{\partial \Lambda} \int_{0}^{1} \mathrm{~d} x[1+\Lambda x]^{-\frac{s}{2}} \\
& =\frac{4}{s \Lambda}-\frac{2}{s \Lambda}\left(1+\Lambda M^{-2}\right)^{-s / 2}+\frac{4}{s(2-s) \Lambda^{2}}\left[1-(1+\Lambda)^{(2-s) / 2}\right] .
\end{aligned}
$$

Likewise

$$
\begin{aligned}
K_{2} \simeq & \int_{M^{-2}}^{\infty} \mathrm{d} x x^{-3 / 2}[1+\Lambda x]^{-\frac{s+2}{2}}=\frac{2 M}{s+3} \frac{M^{s+2}}{\Lambda^{(s+2) / 2}} \\
& \times_{2} F_{1}\left(\frac{s+2}{2}, \frac{s+3}{2} ; \frac{s+5}{2} ; \frac{-M^{2}}{\Lambda}\right) .
\end{aligned}
$$

For Eq. (94) we then obtain approximately

$W(\Lambda, M \ll 1, s) \simeq \frac{M^{2}}{4}\left[\frac{4}{s \Lambda}-\frac{2}{s \Lambda}\left(1+\Lambda M^{-2}\right)^{-s / 2}\right.$

$\left.+\frac{4}{s(2-s) \Lambda^{2}}\left[1-(1+\Lambda)^{(2-s) / 2}\right]\right]$

$+\frac{2 \zeta(s+1)}{\pi(s+3)} \frac{M^{s+2}}{\Lambda^{(s+2) / 2}}{ }_{2} F_{1}\left(\frac{s+2}{2}, \frac{s+3}{2} ; \frac{s+5}{2} ; \frac{-M^{2}}{\Lambda}\right)$ in the limit $\Lambda M^{-2} \gg 1$ we find

$W\left(M^{2} \ll 1, M^{2} \ll \Lambda, s\right) \simeq$

$$
\frac{M^{2}}{s \Lambda}\left[1+\frac{1}{(2-s) \Lambda}\left[1-(1+\Lambda)^{(2-s) / 2}\right]\right]
$$

where we have used the following approximations and relations for the hypergeometric functions (Magnus et al. 1966):

${ }_{2} F_{1}(a, b ; c ; z \ll 1) \approx{ }_{2} F_{1}(a, b ; c ; 0)=1$,

${ }_{2} F_{1}(a, b ; c ; z)=(1-z)^{-a}{ }_{2} F_{1}\left(a, c-b ; c ; \frac{z}{z-1}\right)$,

${ }_{2} F_{1}(a, b ; c ; z)=(1-z){ }_{2}^{-b} F_{1}\left(b, c-a ; c ; \frac{z}{z-1}\right)$,

and if $c>a+b$

${ }_{2} F_{1}(a, b ; c ; 1)=\frac{\Gamma(c) \Gamma(c-a-b)}{\Gamma(c-a) \Gamma(c-b)}$.

B.2. The case of $\Lambda \ll M^{2} \ll 1$

Here we obtain for Eq. (99) approximately

$W\left(\Lambda \ll M^{2} \ll 1\right) \simeq \frac{1}{4}+\frac{2 \zeta(s+1)}{\pi}$

where we have used the same relations and approximations (Eqs. (101)-(104)) as in the last case.

\section{B.3. The case of $1 \ll M^{2} \ll \Lambda$}

In this case $M^{-2} \ll 1$ is a small quantity, and $K_{1}$ is approximately

$K_{1} \simeq 2 \int_{0}^{M^{-2}} \mathrm{~d} x[1+\Lambda x]^{-\frac{s+2}{2}}=\frac{4}{s \Lambda}\left[1-\left(1+\Lambda M^{-2}\right)^{-s / 2}\right]$.

Likewise, $K_{2}$ can be approximated as

$$
\begin{aligned}
K_{2} & \simeq 2 \int_{M^{-2}}^{\infty} \mathrm{d} x x^{-3 / 2}[1+\Lambda x]^{-\frac{s+2}{2}} \\
& -\int_{1}^{\infty} \mathrm{d} x x^{-3 / 2}[1+\Lambda x]^{-\frac{s+2}{2}} \\
& +\int_{0}^{M^{-2}} \mathrm{~d} x x^{-1 / 2}[1+\Lambda x]^{-\frac{s+2}{2}}-\int_{0}^{1} \mathrm{~d} x x^{-1 / 2}[1+\Lambda x]^{-\frac{s+2}{2}} \\
& =\frac{4 M}{s+3} \frac{M^{s+2}}{\Lambda^{(s+2) / 2}}{ }_{2} F_{1}\left(\frac{s+2}{2}, \frac{s+3}{2} ; \frac{s+5}{2} ; \frac{-M^{2}}{\Lambda}\right) \\
& -\frac{2}{s+3} \Lambda^{-(s+2) / 2}{ }_{2} F_{1}\left(\frac{s+2}{2}, \frac{s+3}{2} ; \frac{s+5}{2} ; \frac{-1}{\Lambda}\right) \\
& +\frac{2}{M}{ }_{2} F_{1}\left(\frac{s+2}{2}, \frac{1}{2} ; \frac{3}{2} ;-\Lambda M^{-2}\right)-2{ }_{2} F_{1}\left(\frac{s+2}{2}, \frac{1}{2} ; \frac{3}{2} ;-\Lambda\right) \cdot(107)
\end{aligned}
$$

For Eq. (94) we then obtain approximately

$$
\begin{aligned}
& W(\Lambda, M \gg 1, s) \simeq \frac{M^{2}}{s \Lambda}\left[1-\left(1+\Lambda M^{-2}\right)^{-s / 2}\right] \\
& +\frac{\zeta(s+1)}{\pi M}\left[\frac{4 M}{s+3} \frac{M^{s+2}}{\Lambda^{(s+2) / 2}}{ }_{2} F_{1}\left(\frac{s+2}{2}, \frac{s+3}{2} ; \frac{s+5}{2} ; \frac{-M^{2}}{\Lambda}\right)\right. \\
& -\frac{2}{s+3} \Lambda^{-(s+2) / 2}{ }_{2} F_{1}\left(\frac{s+2}{2}, \frac{s+3}{2} ; \frac{s+5}{2} ; \frac{-1}{\Lambda}\right) \\
& \left.+\frac{2}{M}{ }_{2} F_{1}\left(\frac{s+2}{2}, \frac{1}{2} ; \frac{3}{2} ;-\Lambda M^{-2}\right)-2{ }_{2} F_{1}\left(\frac{s+2}{2}, \frac{1}{2} ; \frac{3}{2} ;-\Lambda\right)\right] .
\end{aligned}
$$


With the transformation formula (Abramowitz \& Stegun 1972)

${ }_{2} F_{1}(a, b ; c ; z)=(1-z)^{c-a-b}{ }_{2} F_{1}(c-a, c-b ; c ; z)$.

Equation (108) reduces to

$$
\begin{aligned}
& W(\Lambda, M \gg 1, s) \simeq \frac{M^{2}}{s \Lambda}\left[1-\left(1+\Lambda M^{-2}\right)^{-s / 2}\right] \\
& +\frac{\zeta(s+1)}{\pi M}\left[\frac{4 M}{s+3} \frac{M^{s+2}}{\Lambda^{(s+2) / 2}}{ }_{2} F_{1}\left(\frac{s+2}{2}, \frac{s+3}{2} ; \frac{s+5}{2} ; \frac{-M^{2}}{\Lambda}\right)\right. \\
& -\frac{2}{s+3} \Lambda^{-(s+2) / 2}{ }_{2} F_{1}\left(\frac{s+2}{2}, \frac{s+3}{2} ; \frac{s+5}{2} ; \frac{-1}{\Lambda}\right) \\
& +\frac{2}{M}\left(1+\Lambda M^{-2}\right)^{-s / 2}{ }_{2} F_{1}\left(\frac{1-s}{2}, 1 ; \frac{3}{2} ;-\Lambda M^{-2}\right) \\
& \left.-2(1+\Lambda)^{-s / 2}{ }_{2} F_{1}\left(\frac{1-s}{2}, 1 ; \frac{3}{2} ;-\Lambda\right)\right]
\end{aligned}
$$

in the limit $\Lambda M^{-2} \gg 1$ and $\Lambda \gg 1$ and with Eq. (101)-(104) we obtain

$$
W\left(1 \ll M^{2} \ll \Lambda\right) \simeq \frac{M^{2}}{s \Lambda}
$$

\section{B.4. The case of $M^{2} \gg 1$ and $M^{2} \gg \Lambda$}

In the last case we obtain for Eq. (108) approximately

$W\left(M^{2} \gg 1, M^{2} \gg \Lambda\right) \simeq \frac{1}{2}+\frac{4 \zeta(s+1)}{\pi}$

where we have used the same relations and approximations as in the first three cases.

\section{References}

Abramowitz, M., \& Stegun, I. A. 1972, Handbook of Mathematical Functions, National Bureau of Standards, Washington

Chandran, B. 2001, Phys. Rev. Lett., 85, 4656

Dung, R., \& Schlickeiser, R. 1990a, A\&A, 237, 504

Dung, R., \& Schlickeiser, R. 1990b, A\&A, 240, 537

Ferriere, K. M., Zweibel, E. G., \& Shull, J. M. 1988, ApJ, 332, 984

Goldreich, P., \& Sridhar, S. 1995, ApJ, 438, 763

Karimabadi, H., Krauss-Varban, D., \& Terasawa, T. 1992, J. Geophys. Res., 97, 13853

Kulsrud, R., \& Perace, W. P. 1969, ApJ, 156, 445

Lerche, I., \& Schlickeiser, R. 2001a, A\&A, 366, 1008

Lerche, I., \& Schlickeiser, R. 2001b, A\&A, 378, 279 (LS)

Lyne, A. G., \& Smith, F. G. 1989, MNRAS, 237, 533

Magnus, W., Oberhettinger, F., \& Soni, R. P. 1966, Formulas and Theorems for the Special Functions of Mathematical Physics (Springer-Verlag Berlin, Heidelberg, New York)

Maurin, D., Donato, F., Taillet, R., \& Salati, P. 2001, ApJ, 555, 585

Minter, A. H., \& Spangler, S. R. 1997, ApJ, 485, 182

Michalek, G., \& Ostrowski, M. 1996, Nonlinear Processes in Geophysics, 3, 66

Rickett, B. J. 1990, ARA\&A, 28, 561

Schlickeiser, R. 1989, ApJ, 336, 264

Schlickeiser, R. 2002, Cosmic Ray Astrophysics (Springer-Verlag, Berlin)

Schlickeiser, R., \& Achatz, U. 1993, J. Plasma Phys., 49, 63

Spangler, S. R. 1991, ApJ, 376, 540

Strong, A. W., \& Moskalenko, I. V. 1998, ApJ, 509, 212

Yan, H., \& Lazarian, A. 2002, Phys. Rev. Lett., in press

Watson, G. N. 1966, A treatise on the Theory of Bessel Functions (Cambridge University Press) 